EMERITA, Revista de Lingüística y Filología Clásica (EM)

LXXVI 1, enero-junio de 2008

pp. $35-53$

ISSN 0013-6662

\title{
FUENTES GRIEGAS EN EL HERBARIO DE PSEUDO-APULEYO: LA CURA DE LA VERBENA CONTRA LA MORDEDURA DEL PERRO RABIOSO*
}

\author{
M. ${ }^{\mathrm{a}}$ Teresa Santamaría Hernández \\ Universidad de Castilla-La Mancha \\ GREEK SOURCES IN THE PSEUDO-APULEIUS HERBAL: \\ THE CURE OF THE VERVAIN AGAINST THE BITE OF THE RABID DOG
}

\begin{abstract}
Este artículo analiza el fragmento del capítulo sobre la herba uerbenaca del Herbario de PseudoApuleyo que ofrece el remedio contra la mordedura de un perro rabioso. Este fragmento es cotejado con otras fuentes médicas griegas y latinas, al tiempo que se revisan manuscritos importantes del Herbario. De este modo hemos detectado adiciones a la sección inicial, que parece ser original, relacionadas con determinadas fuentes griegas, concretamente Oribasio y los escritos toxicológicos de Pseudo-Dioscórides. También obtenemos algunas conclusiones sobre lecturas rechazadas que pueden ser aceptadas, y sobre la presumible existencia de traducciones latinas antiguas de textos médicos griegos que aún no se han atestiguado.
\end{abstract}

This paper analyzes the excerpt from the chapter on the herba uerbenaca of Pseudo-Apuleius' Herbarius offering the cure against the bite of a rabid dog. This excerpt is collated with other Greek and Latin medical sources, while some important manuscripts of the Herbarius are reviewed. Thus, we have detected some additions to the apparently original initial section, which are related to Greek sources, namely Oribasius and Pseudo-Dioscorides' writings on toxicology. We also obtain some conclusions on neglected readings which might be accepted, and on the presumable existence of ancient Latin translations from Greek medical texts which have not yet been attested.

* Este trabajo se inserta en el Proyecto de Investigación HUM2006-13605-C02-01/FILO (Ministerio de Educación y Ciencia). Agradezco a los profesores Arsenio Ferraces Rodríguez, de la Universidad de la Coruña, y Klaus-Dietrich Fischer, de la Universidad de Mainz, la lectura de este trabajo y sus acertadas observaciones 
Palabras clave: Herbario de Pseudo-Apuleyo; fuentes griegas; traducciones latinas; Oribasio; Pseudo-Dioscórides; transmisión manuscrita; crítica textual.
Keywords: Pseudo-Apuleius' Herbarium; Greek sources; Latin translations; Oribasius; Pseudo-Dios corides; manuscript transmission; textual criticism.

Una de las características fundamentales del Herbario de Ps. Apuleyo es su condición de texto elaborado a partir de la incorporación de datos procedentes de diversas fuentes de tipo médico, griegas o latinas, de manera que se puede considerar que parte de su originalidad radica precisamente en aglutinar esos datos siempre con la perspectiva práctica que domina este y otros escritos de la medicina latina tardía. El hecho de que este Herbario fuera un texto práctico facilitó que progresivamente fuera sufriendo alteraciones y adiciones en las distintas curas agrupadas en cada hierba ${ }^{1}$.

Fechado normalmente en su estado más antiguo sobre el siglo IV d.C. y con una amplia y compleja transmisión manuscrita, este texto gozó de una notable difusión en la Edad Media, frecuentemente junto con otros pertenecientes al mismo corpus, como el De taxone liber o el Liber medicinae ex animalibus de Sexto Plácido².

La complejidad del texto, disfrazada en parte por una edición que no refleja el estado real del mismo tal como ha llegado a nosotros ${ }^{3}$, exige todavía hoy un estudio minucioso sobre todo de los fragmentos cuyas fuentes no han sido aún identificadas, como prueba el hecho de que algunos de ellos, de aparente y sintética sencillez, ofrecen de vez en cuando datos sorprendentes y hasta ahora desconocidos.

Precisamente uno de los terrenos todavía no suficientemente explorados es el de las fuentes, sobre las que habría que realizar un estudio que, además

1 Esta circunstancia lleva a M. F. Buffa Giolito a considerar, recogiendo el testimonio de C. Opsomer-Halleux, las interferencias y la contaminación como una ley del género, así como a plantear la necesidad de ofrecer para determinados textos una edición de las distintas versiones existentes. Cf. G. Maggiulli y M. F. Buffa Giolito, L'altro Apuleio. Problema aperti per una nuova edizione dell' Herbarius, Nápoles, 1996, p. 102 y n. 11.

2 E. Howald y H. E. Sigerist (eds.), Antonii Musae de herba vettonica liber. Pseudo Apulei Herbarius. Anonymi de taxone liber. Sexti Placiti liber medicinae ex animalibus, CML IV, Leipzig, 1927. Cf. M. F. Buffa Giolito, «Stato della tradizione dell' Herbarius», en G. Maggiulli y M. F. Buffa Giolito, ob. cit., pp. 98-103.

3 Como ponen de manifiesto G. Maggiulli, ob. cit., p. 34, y A. Ferraces Rodríguez, «Las curae herbarum y las interpolaciones dioscorideas en el Herbario del Pseudo-Apuleyo», Euphrosyne 32, 2004, pp. 223-240, especialmente p. 225. 
de analizar minuciosamente cada fragmento, intente detectar la existencia de aquellas incluso no conservadas y las circunstancias de transmisión de las mismas. Necesario es también en este sentido considerar, junto a las fuentes latinas ya identificadas, la posibilidad de la presencia de originarias fuentes griegas, aunque hayan llegado por la vía de la traducción latina ${ }^{4}$. Esto es especialmente interesante en un texto que, además de la doctrina, tiene también elementos formales en común con la expresión de la medicina de otros textos griegos, anteriores o $\mathrm{no}^{5}$.

Un buen ejemplo de esta situación es el fragmento del Herbario sobre el que realizamos el presente trabajo, la cura Ad canis rabidi morsum et idrofoba de la herba verbenaca (3.9 en la edición de Howald-Sigerist). En esta cura, después de una breve recomendación (Herbam uerbenacam ad uulnus inponito) que se acerca a la estructura básica de otras del Herbario, aunque le faltaría el segmento final con la expresión del resultado del tipo facillime sanat, persanat, etc. ${ }^{6}$, sigue un fragmento más extenso que, lejos de ofrecer las concisas y claras indicaciones que encontramos otras veces, describe un procedimiento que, tal como se presenta, más que para curar la mordedura

${ }^{4}$ Sobre la presencia de Plinio y la Medicina Plinii en el Herbario ha trabajado G. Maggiulli en «Uterque Plinius, uterque (Ps.) Apuleius (Per una ricostruzione della doctrina pliniana nell' Herbarius)», Romanobarbarica 14, 1996/97, pp. 103-142. Por otra parte, según Maggiulli, probablemente circularon entre los siglos IV y VI traducciones latinas de autores griegos que pudieron ser utilizados no sólo ya antes por Plinio o Dioscórides, sino también en el Herbario de Ps. Apuleyo.

${ }_{5}$ Así sucede, por ejemplo, en la cura de la herba brionia (67.1 Ad splenem) en lo que es posiblemente una adición (Haec herba tam laudabilis est, ut in teriacis potionibus mittatur) que recoge la presencia de esta hierba en remedios teriacales para diversos males (Scrib. Larg 165, Gal. XIV K De antidotis II 161, 189, 203; Pr. p. 47, 10, 17-22; Paul. Aeg. 5.3.2) que

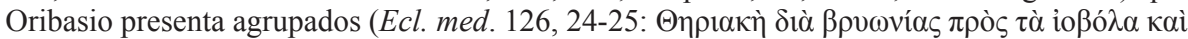

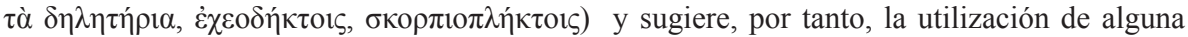
fuente griega, posiblemente no en el momento de la incorporación, sino en un escrito anterior. También el capítulo sobre la hierba basilisca (130 en la edición de Howald-Sigerist) ofrece elementos estructurales y léxicos que lo conectan con la tradición de la toxicología griega, aunque por sus características puede considerarse como extraño a la versión más original del Herbario. De este capítulo me he ocupado en el trabajo «La tradición de la toxicología grecolatina en el Herbario de Ps. Apuleyo: el capítulo sobre la hierba basilisca», Actas del II Seminario Internacional "Textos técnicos grecolatinos», Fito-zooterapia antigua y altomedieval: textos y doctrinas (La Coruña, 21-22 de octubre de 2005), en prensa.

${ }^{6}$ Como explica G. Maggiulli (L'altro Apuleio ..., pp. 39-42), cada cura, que seguiría a un titulus morbi, constaría de tres segmentos correspondientes a planta y parte de la misma utilizada, modo de preparación y administración, y cláusula final con indicación terapéutica y resultados. 
del perro rabioso, sirve para averiguar si ha pasado el peligro. Tratándose de una mordedura de estas características, y teniendo en cuenta algunos datos que luego presentaremos, anticipamos que dicho peligro era el de contraer la hidrofobia que se suponía que afectaba a los atacados si no eran bien cura$\operatorname{dos}^{7}$. El procedimiento consiste, según se explica, en introducir en la herida unos granos de trigo y echarlos después a una gallina para ver si se los come, lo cual indicará que no existe ya riesgo alguno para el enfermo:

\section{Ps. Apul. 3, 9, 30-33}

Herbam uerbenacam ad uulnus inponito, tritici quoque grana integra indito uulneribus, donec humore mollita expleant, iam tumida proicito illa gallinae; si non adpetit, simili modo alia grana coicito; si sic esse coeperit, periculi sublati signum erit.

Sin embargo, junto a la mencionada diferenciación respecto a la que se supone que sería la estructura básica de las curas del Herbario, llaman la atención en esta la referencia a los granos de trigo en un capítulo sobre la verbena - lo que, en principio, podría considerarse un indicio de que se trata de una adición-, y la ausencia de una explicación más detallada del proceso curativo y de la aclaración del peligro de que se trata. Además, HowaldSigerist no ofrecen referencias de posibles fuentes para el contraste, aunque sí lo hacen para otras curas de las doce que presenta esta hierba en su edición.

Con este punto de partida, dos son los recursos que pueden servir para arrojar luz sobre este fragmento: rastrear las fuentes conocidas de la medicina grecolatina a la búsqueda de un procedimiento similar que nos pueda informar sobre su sentido y procedencia, y revisar al menos algunos testimonios importantes de la transmisión manuscrita.

Lo primero nos lleva a descubrir que el experimento de la gallina está presente en las Eclogae medicamentorum que siguen a las Collectiones medicae

7 A esta mordedura y su inclusión en la literatura toxicológica grecolatina he dedicado un capítulo en mi trabajo «La toxicología en los textos médicos latinos de la Antigüedad», Tradición griega y textos médicos latinos en el periodo presalernitano. Actas del VIII Coloquio Internacional «Textos médicos latinos antiguos» (A Coruña, 2-4 de septiembre de 2004), A. Ferraces Rodríguez (ed.), La Coruña, 2007, pp. 289-316. Cf. igualmente F. Gaide, «La rage dans les textes médicaux latins antiques: noms, description, étiologie, traitements», Maladie et maladies dans les textes latins antiques et médiévaux: Actes du V Colloque International «Textes médicaux latins» (Bruxelles, 4-6 septembre 1995), Latomus 242, Bruselas, 1998, pp. 29-41. 
de Oribasio ${ }^{8}$, y después en Aecio y Pablo de Egina ${ }^{10}$, que dice explícitamente haberlo tomado del primero. Reproducimos a continuación los fragmentos correspondientes a los escritos de los tres autores, que, como sabemos, ofrecen importantes semejanzas debidas a su labor de compilación y a la utilización de unos por otros ${ }^{11}$ :

Orib. Ecl. med. 117, 1, 8-16

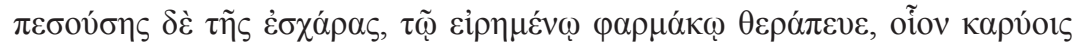

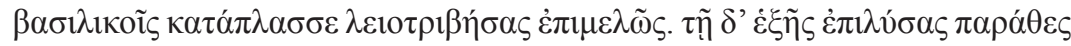

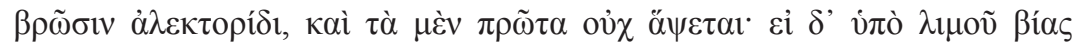

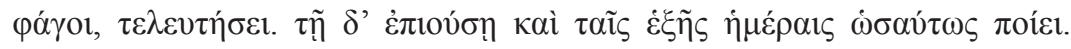

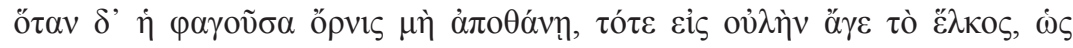

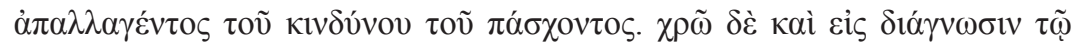

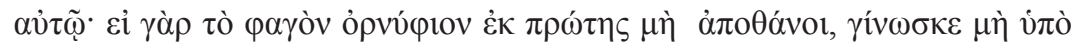

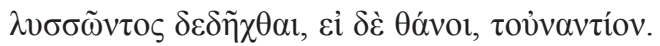

Aët. 6, 24

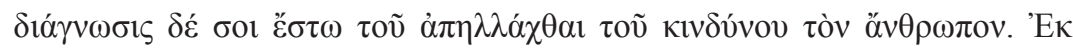

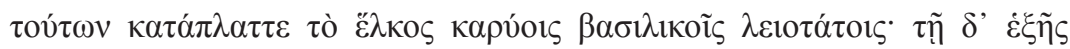

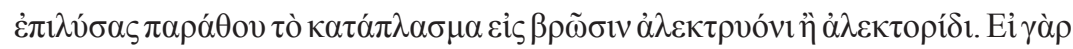

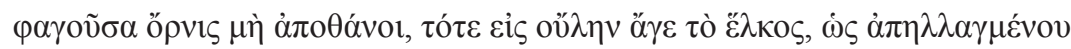

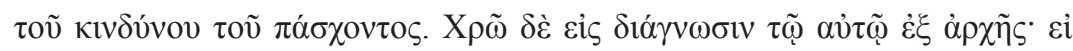

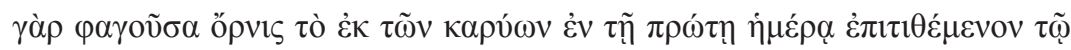

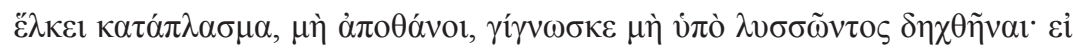

${ }^{8}$ Cf. Oribasii Collectionum medicarum reliquiae. Eclogae medicamentorum (I. Raeder, ed.), $C M G$ VI 2.2, Leipzig-Berlín, 1933. Sobre esta recopilación de remedios, cf. Oeuvres d'Oribase (U. C. Bussemaker, Ch. Daremberg), vol. IV, París, 1862, pp. I-II («Préface»), donde se explica que procederían de libros perdidos de las Collectiones.

9 Hemos manejado la edición renacentista Aetii Amideni librorum medicinalium tomus primus (Venecia, 1534) y la traducción latina de Jano Cornario, Aetii medici Graeci contractae ex veteribus medicinae tetrabiblos ... (Lyon, 1549). Sin embargo, en el fragmento que ofrecemos a continuación seguimos la edición de Rufo de Éfeso de Daremberg-Ruelle, que, como veremos, lo recoge: Oeuvres de Rufus d'Éphèse (Ch. Daremberg, Ch. E. Ruelle, eds.), París, 1879, pp. 371-373.

${ }^{10}$ Pauli Aeginetae Epitomae medicae libri VII (I. L. Heiberg, ed.), vol. 2, CMG IX 2, Leipzig-Berlín, 1924.

${ }^{11}$ Cf. al respecto J.-M. Jacques (ed.), Nicandre. Oeuvres. Les Thériaques. Fragments iologiques antérieurs à Nicandre, París, 2002, pp. XX-XLVIII. 


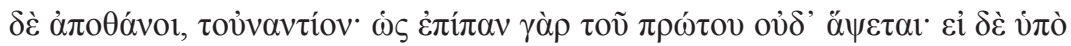

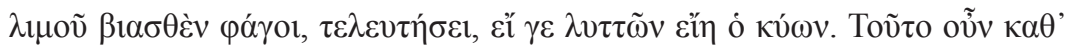

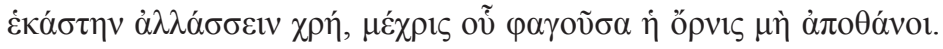

Paul. Aeg. 5, 3, 2

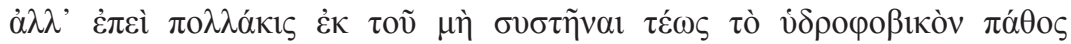

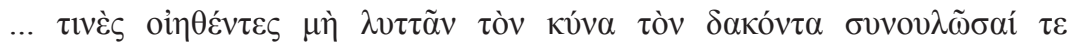

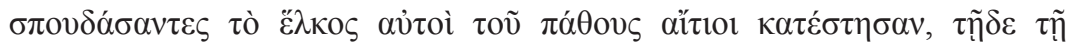

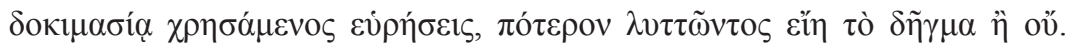

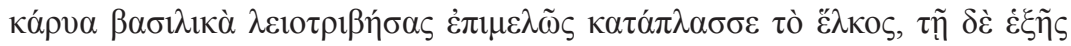

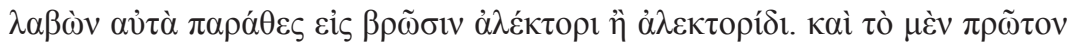

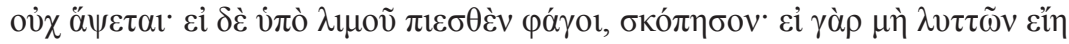

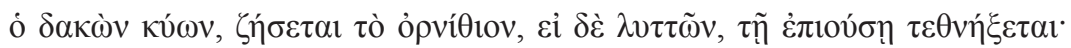

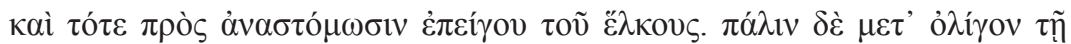

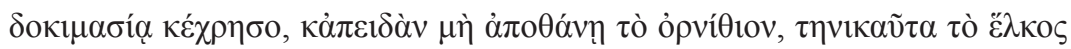

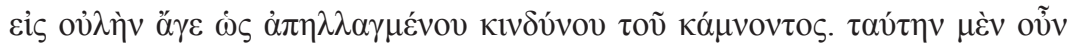

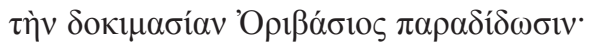

De estos testimonios, el fragmento de Aecio plantea la posibilidad de retrotraer la descripción del experimento a una fuente anterior, pues está incluido en un capítulo que, según se indica en la edición renacentista, está en parte realizado a partir de Rufo de Éfeso. Y efectivamente, el fragmento aparece recogido también en la edición de este autor de Daremberg-Ruelle, aunque en la misma se plantean dudas sobre tal atribución, determinadas entre otras razones porque la tradición no es uniforme en este punto ${ }^{12}$. También encontramos un fragmento similar en el escrito toxicológico de Ps. Elio Promoto, pero la problemática e imprecisa datación del mismo, situado por su editora en el amplio período de los siglos II-VI d.C. ${ }^{13}$, impide plantear conclusiones firmes sobre la cronología de los testimonios y las posibles fuentes, lo cual no significa que no se pueda suponer que haya existido en escritos anteriores,

${ }^{12}$ Oeuvres de Rufus d'Éphèse, ob. cit., pp. 371-375. También son mencionados como fuente del capítulo completo, aunque tampoco de manera uniforme en los testimonios, Galeno y Posidonio.

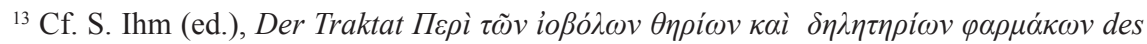
sog. Aelius Promotus, Wiesbaden, 1995, especialmente pp. 17-19, donde la autora ofrece las dataciones defendidas por distintos estudiosos. 
de los que puede haber pasado a estas compilaciones médicas ${ }^{14}$. Éste es el fragmento:

Ps. Ael. Prom. 36

$\delta ı \alpha ́ \gamma v \omega \sigma ı \varsigma \lambda \nu \sigma \sigma o \delta \eta ் \kappa \tau \omega v$

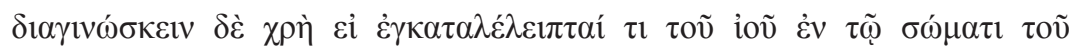

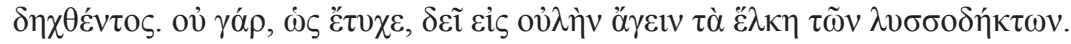

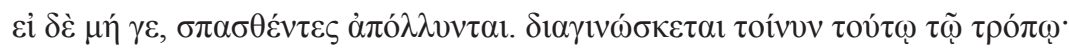

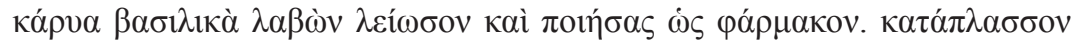

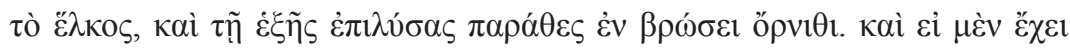

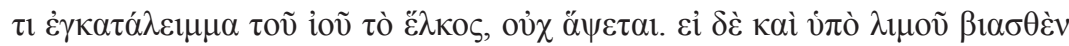

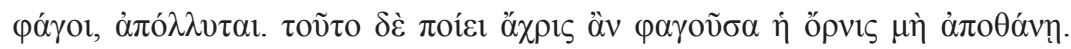

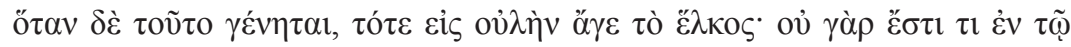

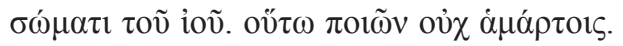

De un contraste inicial del fragmento latino de Ps. Apuleyo con los fragmentos griegos llama la atención, en primer lugar, el hecho de que en aquel se utilicen para este experimento los granos de trigo frente a las nueces

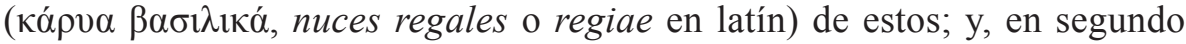
lugar, que la cura del Herbario parece mostrar la simplificación de un procedimiento terapéutico que, según atestiguan los autores griegos, parecía ser más extenso. En efecto, los testimonios griegos ofrecidos, a pesar de sus diferencias ${ }^{15}$, proponen, salvo en el caso de Ps. Promoto, el experimento para una doble finalidad: por un lado, descubrir si ha pasado el peligro de contraer la hidrofobia y se puede proceder a cicatrizar la herida con la seguridad de que esta no retiene ningún resto del veneno que se suponía que inoculaba esta mordedura, algo que indicaba una gallina si no moría al comer los granos procedentes de la herida; $y$, por otro, comprobar si el perro causante del ataque estaba rabioso o no, también en función de la muerte de

${ }^{14}$ La presencia del remedio en Oribasio no excluye que pudiera ser originario de Rufo de Éfeso, que es una de las fuentes que utiliza en las Collectiones medicae, como indica R. De Lucia, «Oribasio di Pergamo», en Medici bizantini (A. Garzya, R. de Lucia et al., eds.), Turín, 2006, p. 28.

${ }^{15}$ Como la expresión más o menos detallada del proceso, alguna inversión en el orden de las fases del mismo, o la mención del veneno en el caso de Ps. Promoto por la perspectiva específicamente toxicológica de su obra. 
la gallina o de su supervivencia. En cambio, el Herbario de Ps. Apuleyo, siempre en la edición de Howald-Sigerist, no recomienda esperar la muerte del animal, sino más bien observar si come los granos o los rechaza, pues el hecho de que los coma indicará que ha pasado el peligro.

Sin embargo, la tradición manuscrita del Herbario, en parte recogida en el aparato crítico de Howald-Sigerist, aporta, si se analizan bien testimonios que en principio podrían parecer confusos, interesantes datos al respecto. En primer lugar, la tradición manuscrita prueba que hay dos redacciones de esta cura, una reducida, a la que es más afín la que ofrecen Howald-Sigerist, y otra más extensa. La primera de ellas está ya atestiguada en el códice de Leiden Vossianus Latinus $Q 9$, que, fechado entre la segunda mitad del siglo VI y principios del VII d.C., es probablemente el manuscrito más antiguo que conservamos con el Herbario de Ps. Apuleyo (rama $\gamma$ de la tradición) ${ }^{16}$, y la ofrecen también testimonios de las ramas $\alpha$ y $\beta$. La redacción ampliada del experimento ofrece notables variaciones con respecto a la primera, visibles sobre todo, aunque no exclusivamente, en la parte final de la misma.

Veamos, en contraste con el fragmento editado por Howald-Sigerist, el testimonio de algunos manuscritos, bien representativos de cada una de las ramas de la transmisión, para las dos redacciones. Para la primera ofrecemos los testimonios de $L 296(\alpha)^{17}$, Laur. 73, $41(\beta)^{18}$ y Voss. Lat. Q9 (Vo, de $\left.\gamma\right)$. Para la segunda redacción presentamos las versiones que de ella ofrecen los manuscritos Vratislaviensis III F $19(\mathrm{Vr})$, que recoge, como aclararemos más

${ }^{16} \mathrm{Cf}$. al respecto E. Howald, H. E. Sigerist (eds.), Antonii Musae de herba vettonica liber. Pseudo Apulei Herbarius ..., ob. cit., p. XIII; M. F. Buffa Giolito, «Stato della tradizione dell' Herbarius», L'altro Apuleio ..., ob. cit., p. 98 y nota 3.

${ }^{17}$ Ms. de Lucca, Biblioteca Statale, L 296, siglo IX. Sobre él, cf. E. Howald, H. E. Sigerist (eds.), Antonii Musae de herba vettonica liber. Pseudo Apulei Herbarius ..., ob. cit., p. VII; A. Beccaria, I codici di medicina del periodo presalernitano (secoli IX, X e XI), Roma, 1956, pp. 285-288; A. Mancini, «Pseudo Apulei libellum de medicaminibus herbarum ex codice Lucensi 296», Atti della Reale Academia Lucchese di scienze, lettere ed arti, 32 (1904), pp. 253-301. El otro manuscrito importante de esta rama, el Casinensis 97, no ofrece las curas de la verbena. Cf. A. Beccaria, ob. cit., pp. 297-303.

${ }^{18}$ Florencia, Biblioteca Medicea Laurenziana, siglo IX. Cf. A. Beccaria, I codici di medicina ..., ob. cit., pp. 281-284; y M. F. Buffa Giolito, «Il Laur. pl. LXXIII.cod. 41», L'altro Apuleio ..., ob. cit., pp. 104-111. Este manuscrito, prácticamente ignorado por Howald-Sigerist, ha sido estudiado y valorado por esta autora, que lo considera relevante en la tradición manuscrita del Herbario. De su estudio tomamos la versión que ofrece este ejemplar de la cura de la verbena que nos ocupa (p. 129). 
adelante, las dos redacciones ${ }^{19}$, y Vindobonensis 93 (Vi), ambos de la rama $\beta^{20}$. Omitimos aquellas lecturas de fragmentos de todos estos manuscritos que coinciden con las ofrecidas por Howald-Sigerist $(=H-S)$ en su edición.

Ad canis rabidi morsum et idrofoba $H-S$ rauidi Laur hidrofoba $V o$ hydrofobum $L$ ydrofoba $V i$

Herbam uerbenacam $H$-S Herba uerminacia Vo Laur Herba uermenacem $L$ Herbam uerminaciam $\operatorname{Vr}$ Herba uerminatia $V i$

ad uulnus $H-S$ in uulnus Laur super morsum canis rabiosi $V i$

inponito $H-S$ imponito $L$ inponis $L a u r$ ad ponis $V r$ ponis $V i$

tritici quoque $H-S$ et tritici quoque $\mathrm{Vr}$

grana integra $H-S$ grana $o m$. Vo grana undecim Laur grana integra XIII aut XV $\mathrm{Vr}$ grana integra XIII aut XV Vi

indito (corr. $H-S$ ) uulneribus $H$-S adpositum uulneribus $V o$ inditum uulneri $L$ adposita uulneri Laur adposita uulneribus $V r$ super uulnus $V i$

humore mollita $H-S$ umore mollita $V o$ humori remollita $L$ cum corrire mollita $\mathrm{Vi}$

expleant $H-S$ se repleant Laur expleantur $V r$ repleantur $V i$

iam tumida $H$-S tumidas $V o$ iam tumidas $L$ et tumida Laur $\mathrm{Vr} V i$

proicito illa ${ }^{21}$ gallinae $H-S$ tollito et prohicito illas galline $V o$ proicito illas gallinae $L$ Laur $V r$ proicies illas galline $V i$

\footnotetext{
${ }^{19}$ Utilizamos cursiva para las adiciones que en $V r$ incorporan la redacción amplia a la reducida. Sobre las adiciones y correcciones de este manuscrito, cf. A. Beccaria, I codici di medicina ..., p. 341.

${ }^{20}$ Respectivamente, Breslau, Biblioteca Universitaria, siglo IX, y Viena, Österreichische Nationalbibliothek, siglo XIII. Cf. E. Howald y H. E. Sigerist (eds.), Antonii Musae de herba vettonica liber. Pseudo Apulei Herbarius ..., pp. X-XI; A. Beccaria, I codici di medicina ..., pp. 341-343, para $V r$.

${ }^{21}$ M. F. Buffa Giolito (L'altro Apuleio ..., p. 130) considera illa una corrección de Howald-Sigerist, quizá porque no aparece en los testimonios que los editores dicen utilizar en el capítulo de la verbena: $\alpha$ : $L$ ( $H a V), \beta: V r V i, \gamma: V o$. Sin embargo, otros testimonios de la transmisión manuscrita, como Harleianus 5294 o Parisinus Arsenal 1031 (clase $\beta$ ), ofrecen esta lectura. Por otra parte, Howald-Sigerist no han recogido en su aparato crítico la lectura illas, que sí ofrecen, por ejemplo, $L$ y Vo. También encontramos tumidas e illas en la exposición de esta cura que recogen en el capítulo correspondiente a la verbena las Curae herbarum, compilación fitoterapéutica que cuenta entre sus fuentes con el Herbario de Ps. Apuleyo y que hemos consultado en los manuscritos L 296 y Londres, Wellcome Medical Library, 573 (s. XIII). Acerca de esta compilación y las fuentes que utiliza, cf. A. Ferraces Rodríguez, Estudios sobre textos latinos de fitoterapia entre la Antigüedad Tardía y la Alta Edad Media, La Coruña, 1999, pp. 141-152.
} 
si non adpetit, simili modo alia grana coicito $H-S$ si non adpetens $L$ si non adpetens fuerit Laur $V r$ si appetens fuerit $V i$ coiicito $V o$ conicito $V r$

si sic esse coeperit $H-S$ coeperint $V o$ ceperit $L^{e t}$ si esse coeperint / si ea tulerit $\mathrm{Vr}$ si eos tolent $\mathrm{Vi}$

periculi sublati (corr. $H-S$ ) signum erit $H-S$ periculosum signum erit $V o$ pericula sublati signum erit $L$ periculosum et mortiferum signum erit Laur utilissimum erit $\mathrm{Vr}$ Vi

si autem appetens non fuerit periculosum signum et mortiferum erit $\mathrm{Vr} V i$

Para completar la exposición de los hechos y mostrar más claramente las dos redacciones, sobre todo en lo relativo a la parte final, presentamos independientemente, a modo de ejemplo, un testimonio de cada una que pueda servir de guía en el presente trabajo, teniendo en cuenta las variantes recogidas arriba. Para la redacción breve del experimento tomamos la de $L$, y para la extensa ofrecemos la versión de $\operatorname{Vr}$ (que - recordemos - incluye las dos), destacando en cursiva los términos incorporados al margen y recogiendo entre paréntesis la versión reducida, que es la que se encuentra en el cuerpo principal del texto:

$L$

herba uermenacem ad uulnus imponito; tritici quoque grana integra inditum uulneri, donec humori remollita expleant. Iam tumidas proicito illas gallinae; si non adpetens, simili modo alia grana coicito; si sic esse ceperit, pericula sublati signum erit.

$V r$ $\mathrm{p}$

. XIII aut XV

Herbam uerminaciam ad uulnus adponis; et tritici quoque grana integra . adposita uulneribus, donec humore mollita expleantur; et tumida proicito illas gallinae

$\mathrm{p}$

.et

si non adpetens fuerit, simili modo alia grana conicito. si ea tulerit, utilissimum erit; si autem appetens non fuerit, periculosum signum et mortiferum erit. (si esse coeperint, periculosum signum et mortiferum erit). 
Una vez establecidas las variantes del texto, el análisis de las mismas nos conduce a las siguientes observaciones:

\section{1. tritici quoque grana ... expleant $(H-S)$}

Junto a la cura inicial, más breve y propia específicamente de la verbena (Herbam uerbenacam ad uulnus inponito), se introduce un remedio distinto que probablemente no pertenecería al capítulo original, sino que habría sido añadido después. A ello apuntan no sólo la presencia de un ingrediente diferente (los granos de trigo), sino también el adverbio quoque, que marca la adición de otro remedio. Admitiendo que se trata de una cura distinta, dos son las interpretaciones posibles de este fragmento. Como prueban los testimonios aducidos de la medicina griega, era tradicional un experimento similar que se realizaba con nuces regiae cuidadosamente trituradas (Orib.

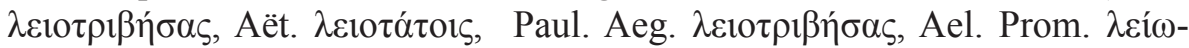
$\sigma o v)$. A este respecto, podría pensarse que un posible trita hubiera sido sustituido, ya en fecha temprana, por el término tritici que ofrecen los testimonios de la transmisión. A esto podría apuntar la presencia de trita en un remedio similar recogido en el Botanicus de St. Gall, que ofrece una reelaboración diferente de la primera parte del remedio $^{22}$ :

\subsection{Ad canes rapidem morsum}

Herba uerminaca ad ulnus trita inposita donec humores remolestiat, uenenum extrait; de ipsa grana facis expormento: quem de ipsa plaga tollis, das ei pullo commedere; sed ipsa herba grana comederit, periculum mortis significat.

La editora del Botanicus, sin embargo, plantea la posibilidad contraria, es decir, que desde un original triticum el compilador prefirió tritus, posiblemente porque le resultaba más familiar en un recetario ${ }^{23}$.

Y, en efecto, a la defensa de la presencia original de tritici en esta cura apunta otro testimonio de la medicina griega, hasta ahora no tenido en cuenta

${ }^{22}$ Cf. M. Niederer (ed.), Der St. Galler Botanicus. Ein frühmittelalterliches Herbar, Berna, 2005. Según Niederer, el Botanicus, conservado en un manuscrito del siglo IX, utiliza frecuentemente como fuente el Herbario de Ps. Apuleyo.

${ }^{23}$ Cf. M. Niederer (ed.), Der St. Galler Botanicus ..., pp. 225-226. 
para la explicación de este fragmento. Si se repasa minuciosamente en la literatura toxicológica antigua el tratamiento recomendado para la mordedura del perro rabioso, se descubre que uno de los escritos toxicológicos de Ps. Dioscórides recogía el empleo de los granos de trigo en la curación de las heridas provocadas por este animal, recomendándolo para dilatar la herida ${ }^{24}$ :

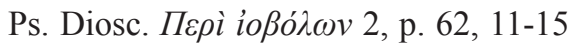

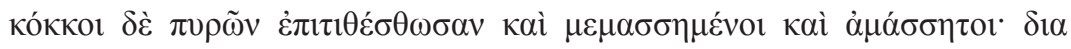

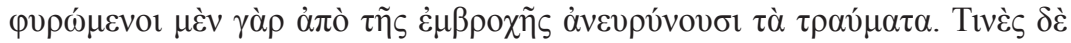

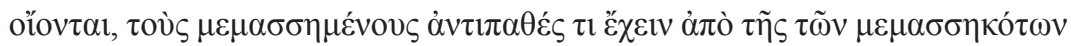

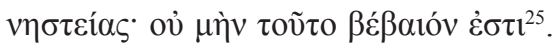

Como puede comprobarse, aunque la segunda parte no coincide en absoluto con la descripción de Ps. Apuleyo, sí hay una evidente conexión con la primera parte, donde se observan trazos evidentes de traducción:

Tritici quoque grana

Adpositum /-a / inditum

Humore (re)mollita

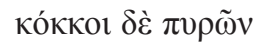

$\dot{\varepsilon} \pi \imath \tau \imath \varepsilon \dot{\varepsilon} \sigma \theta \omega \sigma \alpha \nu$

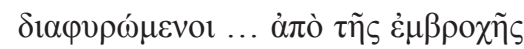

Este hecho constituye un importante dato para apoyar la idea de que estamos ante un remedio diferente, como prueba, además, no ya la presencia de los granos de trigo, sino también de humore, que no aparecía en los fragmentos antes ofrecidos sobre el experimento de las nueces. Además, la relación con este texto griego puede llevarnos a defender, en tanto que parecen traducciones más atinadas, las más cercanas lecturas de indit- y expleant, esta última en el sentido de 'llenar la herida', más aproximado a ảvevpv́vovбı frente a se repleant o relexpleantur de otros testimonios ${ }^{26}$. Incluso podría

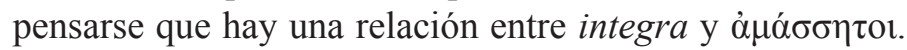

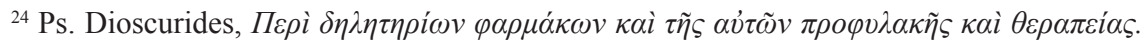

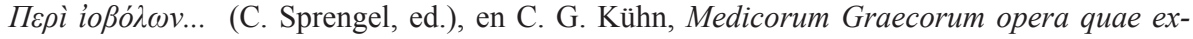
stant, vol. 26, tomo 2, Leipzig, 1830, pp. 1-91.

${ }^{25}$ Remedio parecido encontramos en Plinio a propósito de las nueces: HN XXIII 149 Contra rabiosi quoque canis morsum hi nuclei a ieiuno homine commanducati inlitique praesenti remedio esse dicuntur.

${ }^{26}$ Frente a lo que prefiere M. F. Buffa Giolito ( $L$ 'altro Apuleio ..., ob. cit., p. 130), que se decanta por la lectura se repleant de Laur. 73.41 y otros manuscritos, argumentando, entre 
2. iam tumida ... signum erit $(H-S)$. Las dos redacciones

Esta parte del fragmento remite evidentemente al experimento de las nueces transmitido por las fuentes griegas. El contraste con las mismas nos revela los siguientes datos:

En primer lugar, si recordamos que en los fragmentos griegos el procedi-

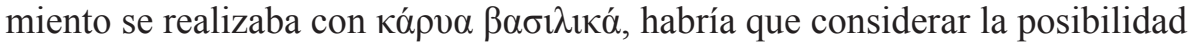
de aceptar, como propia de una traducción latina original más afín al texto griego, la lectura illas atestiguada abundantemente en la tradición manuscrita desde $V o$, pero totalmente ignorada por Howald-Sigerist. En efecto, ese término illas (y tumidas en Vo y L 296) podría estar referido a unas nuces regiae o regales, denominación latina para кápva $\beta \alpha \sigma \lambda \lambda \iota \kappa \alpha$, y de género femenino ${ }^{27}$.

También la relación con una redacción griega original puede arrojar luz sobre el sentido de la frase si sic esse coeperit. A este respecto, a propósito de la redacción que ofrece Laur. 43, 71, M. F. Buffa Giolito justificaba esta frase en conexión con la anterior si non adpetens fuerit, cuyo término adpetens habría que entender aquí: si sic (= adpetens) esse coeperit. Sin embargo, la valoración de la redacción griega, y sobre todo la aparición en ella de las

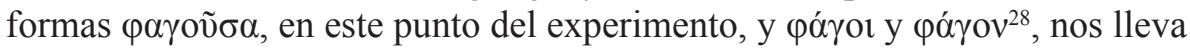
a descartar la necesidad de entender adpetens o similar, y a interpretar esse no como infinitivo de sum, sino de edo. También el Botanicus (sed ipsa herba grana comederit ...) apoya esta afirmación.

En cuanto a las dos redacciones del experimento, una más breve y otra más extensa y detallada, ambas son coherentes en sentido con la tradición griega, y vienen a apoyar la idea de que en algún momento pudieron circular varias reelaboraciones de una traducción latina inicial o incluso previas a la misma y todavía en griego. Esto es lo que pueden indicar, además de las variantes de la tradición manuscrita de Ps. Apuleyo, las que encontramos en el Botanicus. Este escrito presenta, en la versión reducida, un texto con afinidades al que ofrecen las fuentes griegas, y diferencias con respecto a

otras razones, que expleant quedaría sin objeto expreso. Efectivamente, aquí no lo tendría, pero sí podría haber estado presente en el texto griego original y, quizá, en una traducción o reelaboración latina anterior a este estado de texto que nos ofrece el Herbario de Ps. Apuleyo.

${ }^{27}$ Cf. J. André, Les noms de plantes dans la Rome antique, París, 1985, pp. 172-173.

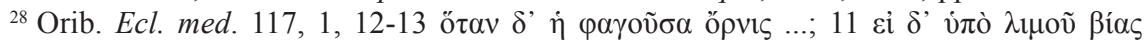

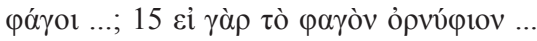


Ps. Apuleyo: además de la aparición del término expormento (por experi-

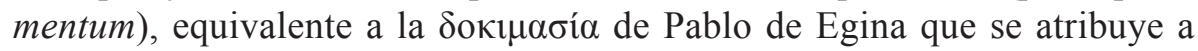
Oribasio $^{29}$, tiene fragmentos de traducción que no encontramos en el Herbario latino, relativamente apegados al texto griego ${ }^{30}$.

Bot. 6, 6 quem de ipsa plaga tollis, das ei ad pullo commedere

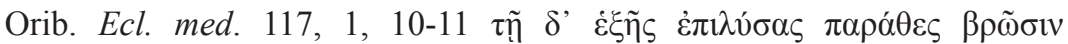
$\dot{\alpha} \lambda \varepsilon \kappa \tau$ opí $\delta$.

Bot. sed ipsa herba grana comederit, periculum mortis significat

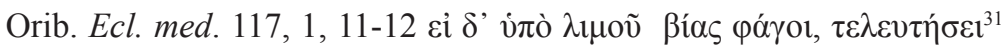

El fragmento de Ps. Apuleyo, tomado ahora tal como lo editan Howald y Sigerist, y particularmente con el final — corregido- de la redacción breve que encontramos en $L$, ofrece también una importante relación con algunos fragmentos de la versión griega, aunque, como hemos visto, esté reducida a

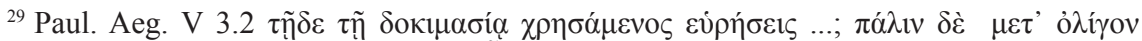

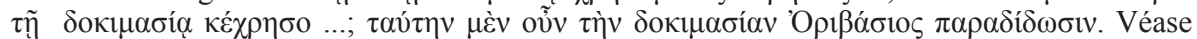

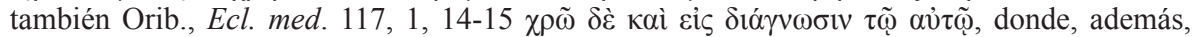
se nos plantea si no habrá que ver una equivalencia entre ipsa (Bot. de ipsa grana facis

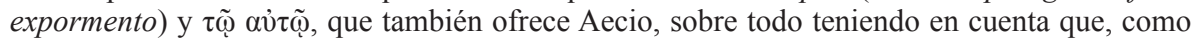
indica M. Niederer (ob. cit., p. 226), ese grana ha podido ser añadido por el compilador, llevado por la lectura en la supuesta fuente de los tritici grana del fragmento anterior en Ps. Apuleyo.

${ }^{30}$ Estas afinidades, junto con algunos hechos, como que Aët., en el estado en que lo conocemos, presente un orden diferente en las fases del procedimiento o Paul. Aeg. ofrezca

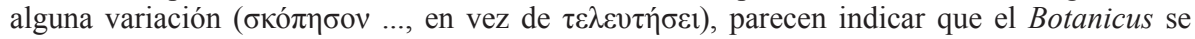
acerca más al texto de Oribasio.

${ }^{31}$ Otro testimonio del experimento, aunque merecedor de una valoración distinta, es el que encontramos en el Canon de Avicena, que, en este caso concreto, parece combinar dos versiones similares a las que ofrecen, respectivamente, Ps. Apuleyo y Oribasio: Canon IV, fen 6, tract. 4 (Differentia inter morsum canis rabiosi, et non rabiosi) Quandoque mordet homines canis, et non aduenit affirmatio formae eius, et verificatio dispositionum eius et indiget curatione eius. Et curatio quidem eius inquantum est vulnus, est consolidatio: et inquantum est morsus canis rabiosi, est saniositas et apertio. Nam si consolidatur, est in ea perditio: quare indiget illud signo, ex quo eius dispositio cognoscatur. Et ex eis, que dixerunt in hoc, est sumatur nux regalis aut alia, et ponatur super vulnus: et dimittatur super ipsum una hora, deinde sumatur, et proiiciatur gallinae: tunc si renuit eam, canis est rabiosus: et si comedit eam, et moritur, tunc canis iterum est rabiosus. Hemos utilizado la edición del Canon de Avicena de Venecia, 1608, con la traducción de Gerardo de Cremona y la revisión de Andrés Alpago. 
lo esencial y carezca de toda la explicación sobre la cicatrización de la herida. Contrastemos con el texto de Oribasio:

iam tumida proicito illa gallinae si non adpetit, simili modo alia grana coicito

si sic esse coeperit, periculi sublati signum erit

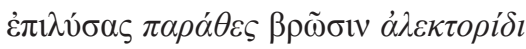

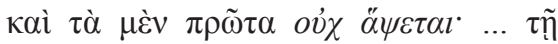

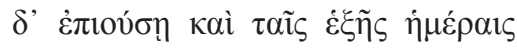

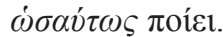

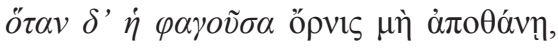

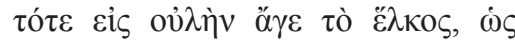

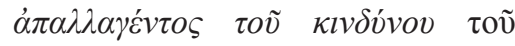
$\pi \alpha ́ \sigma \chi 0 \nu \tau o \varsigma$.

Sobre esta redacción de Ps. Apuleyo podemos hacer varias aclaraciones. Al menos en el estado en que lo conservamos, el fragmento latino parece conectar bien con el texto de Oribasio, no ya porque mantiene el mismo orden en la exposición de los hechos, pues este orden puede haberse alterado en los otros textos en algún momento de la transmisión, sino sobre todo por algunas coincidencias léxicas exclusivas de este texto griego. Me refiero, en concreto, a la secuencia simili modo, equivalente del griego $\omega \sigma \alpha v ́ \tau \omega \varsigma$, y a la presencia exclusiva de la gallina ( $\dot{\alpha} \lambda \varepsilon \kappa \tau$ opís, también denominada con ópvis y ópvú(ııv), y no del gallo, que sí aparece en los restantes fragmentos griegos, como instrumento del experimento. En este punto conviene recordar que, probablemente, el término pullo que ofrece el Botanicus en el experimento proceda de una de las variantes de traducción a que antes aludíamos ${ }^{32}$.

Por otra parte, la alusión al periculi ( $-a L)$ sublati, evidente traducción de

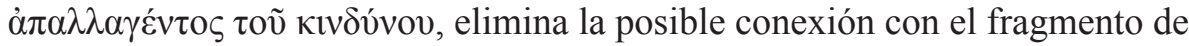
la tradición específicamente toxicológica que representa Ps. Promoto, donde no aparecía.

Precisamente en cuanto al resultado final del experimento, los testimonios analizados nos ofrecen en la redacción reducida dos valoraciones distintas. El contraste con las fuentes griegas sirve igualmente para explicar

${ }^{32}$ M. Niederer, ob. cit., pp. 227-228, explica la utilización de pullus por gallina como un rasgo de latín vulgar, pero puede plantearse también, en la línea de lo que venimos comentando, que su presencia en este fragmento del Botanicus esté determinada por la aparición del término (Orib. ỏpvúpıv, Paul. Aeg. ỏpví⿴囗ov) en un original griego. 
la existencia en la tradición manuscrita de fragmentos aparentemente contradictorios, como un final del tipo periculosum et mortiferum signum erit (o periculosum signum erit), frente a periculi sublati signum erit, y comprobar que no hay tales contradicciones, como un primer acercamiento a las dos versiones podría plantear. Por un lado, se recoge la idea de que ha pasado el peligro, como permite sostener la lectura de L 296 (si sic esse ceperit pericula sublati signum erit), que se corresponde perfectamente con el texto griego. Pero, por otro lado, también encontramos ampliamente atestiguada ya desde $\operatorname{Vo}$ (Laur 73, 41, Barb. 160, Harl. 529433) la versión contraria, que podría responder igualmente a otro fragmento de la descripción griega del experimento ${ }^{34}$ :

Laur 73. 41 si sic esse coeperit, periculosum et mortiferum signum erit (et mortiferum om. Vo)

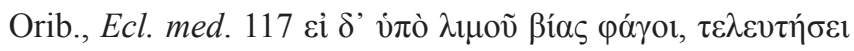

Además, esta versión coincide también con la que presentan el Botanicus, si bien con una traducción diferente (sed de ipsa herba grana comederit, periculum mortis significat), y el Canon de Avicena (et si comedit eam, et moritur, tunc canis iterum est rabiosus).

Por otra parte, en esta sección final se desarrolla también, como hemos visto, en algunos manuscritos una descripción más detallada del procedimiento, que, recogiendo igualmente el significado y las fases del mismo, sin embargo presenta una menor correspondencia formal. En efecto, frente al apego al texto griego de la redacción reducida, la redacción amplia parece ser una reelaboración de contenidos a partir del significado del experimento original, lo cual no significa que no sea coherente en lo que dice, que es acorde también con el contenido del texto griego. Esta redacción habría aparecido probablemente para explicar más detenidamente el experimento, aunque no podamos saber si de manera independiente de una versión breve o a partir de ella.

\footnotetext{
${ }^{33}$ Sobre estos manuscritos, cf. E. Howald y H. E. Sigerist (eds.), Antonii Musae de herba vettonica liber. Pseudo Apulei Herbarius ..., p. XII.

${ }^{34}$ Aunque no se pueda descartar que se deba a una interpretación distinta de algún término o a otras circunstancias de la transmisión.
} 


\section{Conclusiones}

En esta cura de la verbena, el Herbario de Ps. Apuleyo, tal como ha llegado a nosotros, ofrece un fragmento que parece responder a lo que en un momento anterior habrían sido tres remedios distintos, aunque pueda pensarse que aquí están más o menos integrados para dar coherencia al capítulo. Esta afirmación se ve apoyada por la redacción del Botanicus, que, además de distinguir explícitamente dos partes ${ }^{35}$, utiliza, por ejemplo, los términos ulnus (en lugar de la forma uulnus que aparece en Ps. Apuleyo) y plaga para las dos secciones del capítulo, lo que podría apuntar a un origen diferente de ambos fragmentos.

Probablemente, frente a la sección inicial (Herbam uerbenacam ad uulnus inponito), el resto hayan sido añadidos en un momento posterior a la redacción primera del Herbario, lo que explica que el capítulo no responda a la estructura básica, más simple, a la que respondería en un primer momento.

El origen de estos remedios diferentes para la mordedura del perro rabioso, y la razón de que estén aquí reunidos, es que, probablemente, figuraban agrupados así en alguna colección de excerpta, sin que podamos saber si la reelaboración a que aquí están sometidos se debió a quien los incorporó al Herbario o fue realizada en un momento anterior.

En cualquier caso, tanto el fragmento del trigo como el de la gallina parecen conectar con la tradición y los textos de la medicina griega. El primero

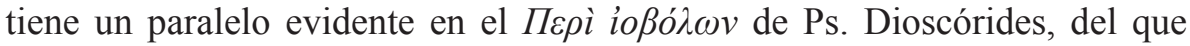
bien podría ser una traducción parcial. En cuanto al segundo, ofrece seguramente restos de traducción latina de la redacción griega de un experimento muy tradicional, como prueba su aparición, entre otros, en los escritos de Oribasio, Aecio y Pablo de Egina.

A este respecto, los fragmentos más afines al texto griego se encuentran en $V o$, de la clase $\gamma$, para la primera parte (tollito et prohicito) y en $L 296$, de $\alpha$, (pericula sublati signum erit), que revelan una innegable correspondencia entre ambas tradiciones.

Por otra parte, aunque lo que recoge el Herbario de Ps. Apuleyo tiene un carácter parcial o fragmentario, sin una exposición detallada de los pasos del

\footnotetext{
${ }^{35}$ Como expresan claramente los fragmentos uenenum extrait, que cerraría la inicial (Herba uerminaca ad ulnus trita inposita donec humores remolestiat, uenenum extrait), que aglutinaría además las dos primeras de Ps. Apuleyo, y de ipsa grana facis expormento, que introduce el procedimiento de la segunda parte.
} 
experimento como lo ofrecen los textos griegos, las variantes que la tradición manuscrita del Herbario ofrece en la redacción de la última parte nos hacen pensar que, probablemente pudieron haber circulado una o varias versiones latinas más amplias del mismo, a lo que apunta, por ejemplo, la existencia en el Botanicus de términos y fragmentos no presentes en Ps. Apuleyo (por ejemplo expormento).

En este sentido, el testimonio del Botanicus, donde se conserva también el experimento, ofrece lo que parece ser una traducción con un léxico distinto (plaga, commedere, pullus, frente a uulnus, esse, gallina) y con un apego mayor al texto griego en algún punto (das ei ad pullo commedere).

La posibilidad de admitir un origen griego para estos fragmentos podría llevar a pensar en la existencia de traducciones latinas de las obras que los recogen. Así, el fragmento de los tritici grana podría proceder de una traducción latina —al menos parcial— del Ps. Dioscórides o de una fuente común, aunque también se podría pensar en la incorporación directa a una colección de remedios.

En cuanto al fragmento de la gallina, es muy tentadora la idea de ver aquí restos de una traducción de los escritos de Oribasio de los que no hay atestiguada versión latina alguna. En efecto, hasta ahora se ha venido sosteniendo que sólo existieron las de la Synopsis y los Euporista, pero no de las Collectiones medicae o de fragmentos relacionados con ellas ${ }^{36}$. Algunos elementos léxicos del fragmento de Ps. Apuleyo (simili modo, gallinae), conectados con términos que solo aparecen en la versión de Oribasio, así lo apuntan, y no es descabellado pensar en la existencia de esta traducción, total o parcial, cuando fueron también traducidas sus otras obras ${ }^{37}$. Una cuestión bien distinta es poder precisar cuándo entraron en el Herbario esos restos.

Por último, la identificación de posibles fuentes o, al menos, de textos griegos relacionados en forma y contenido con el latino nos lleva a defender

${ }^{36}$ Cf. H. Mørland, Die Lateinischen Oribasiusübersetzungen, Oslo, 1932; M. E. Vázquez Buján, «Problemas generales de las antiguas traducciones médicas latinas», Studi medievali 25, 1984, pp. 641-680; G. Sabbah et al. (dirs.), Bibliographie des textes médicaux latins. Antiquité et Aut. Moyen Âge, Saint-Étienne, 1987, p. 120.

${ }^{37}$ A ello apunta también la localización por A. Ferraces Rodríguez de un fragmento latino de las Collectiones medicae en la Diaeta Theodori. Véase su trabajo «Oribasiana», en La transmisión de la Ciencia desde la Antigüedad al Renacimiento (M. a T. Santamaría Hernández, ed.), Cuenca, 2008, pp. 71-95, especialmente 80-84. 
la cautela a la hora de elegir determinadas lecturas frente a otras consideradas erróneas. En textos donde la contaminación ocupa un papel fundamental como procedimiento creativo, quizá no haya que buscar siempre la coherencia en el resultado final que analizamos, sino más bien en estados anteriores al mismo ${ }^{38}$. Determinadas lecturas pueden ser, en relación con los textos originales de los que proceden, totalmente aceptables: ¿illa o illas?

Fecha de recepción de la primera versión del artículo: 31/05/2007

Fecha de recepción de la versión definitiva del artículo: 11/06/2008

Fecha de aceptación del artículo: 18/06/2008

${ }^{38}$ Cf. al respecto A. Ferraces Rodríguez, «Correcciones indebidas en las Etimologías de Isidoro de Sevilla», La Filología Latina hoy: actualización y perspectivas (A. Ma. Aldama et al., eds.), vol. I, Madrid, 1999, pp. 649-660, que plantea con gran acierto, a propósito del escrito isidoriano, la necesidad de analizar las fuentes conservadas total o parcialmente a la hora de resolver problemas textuales. 